\title{
A COMPUTER MODEL FOR SINGLE-BREATH WASHOUT WITH DOUBLE-TRACER GAS TO HELP UNDERSTANDING VENTILATION INHOMOGENEITY
}

\author{
David Borer ${ }^{1}$, Sophie Yammine ${ }^{2}$, Florian Singer ${ }^{2}$, Thomas Rösgen ${ }^{1}$, Philipp Latzin ${ }^{2}$ and Dominik Obrist ${ }^{1}$ \\ ${ }^{1}$ Institute of Fluiddynamics, ETH Zürich, Switzerland \\ ${ }^{2}$ Division of Respiratory Medicine, Dept of Paediatrics, University Hospital of Bern, Switzerland
}

dborer@ifd.mavt.ethz.ch

\begin{abstract}
We present a numerical model for a single-breath washout of a helium-sulfur hexafluoride-air mixture. The model is validated against measured data.
\end{abstract}

Keywords: Single breath washout, double tracer gas, cystic fibrosis, numerical model.

\section{Introduction}

In lung disease, tests for ventilation inhomogeneity over several breaths (multiple-breath washout) are sensitive but not established for clinical routine due to long duration. A new test over one breath (single-breath washout; SBW) has recently been invented. This test uses a gas mixture containing $5 \%$ sulfur hexafluoride and $26.3 \%$ helium and is thus called double-tracer gas (DTG) SBW. It is easy to perform in children and seems promising for clinical routine due to the short duration. Children with cystic fibrosis (CF) lung disease have steeper slopes of the signal during phase III of the exhalation (SIII) compared to healthy controls [1].
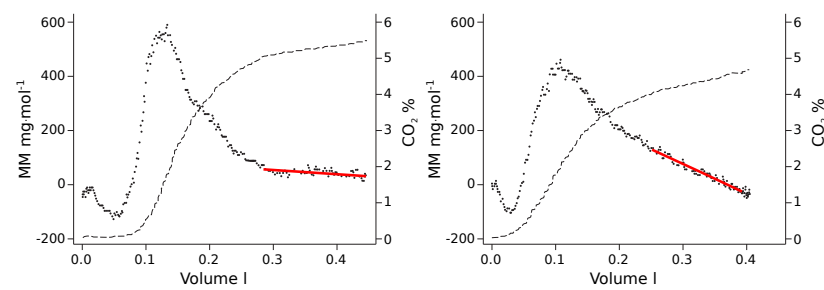

Figure 1: DTG-SBW giving molar mass (MM) samples (dots) and carbon dioxide (CO2) samples (dashes) plotted against expired volume in a boy with $\mathrm{CF}$ (right panel) and a healthy boy (left panel), both aged nine years. Following $\mathrm{MM}$ correction for the $\mathrm{CO} 2$ fraction, $\mathrm{MM}$ above zero reflects greater SF6 than He contribution and, vice versa, MM below zero reflects greater He than SF6 contribution. Investigators fitted the slope (SIII, solid line) according to the phase III of MM and CO2 between $65 \%$ and $95 \%$ of expired volume. SIII was $-591.6 \mathrm{mg} / \mathrm{mol} / \mathrm{L}$ in $\mathrm{CF}$ and -65.8 $\mathrm{mg} / \mathrm{mol} / \mathrm{L}$ in healthy control.

We have developed a computer model for the DTG-SBW tests which simulates the ventilation of the whole lung with the different gas species. The predicted composition of the exhaled air can be directly compared to the measured MM signal from a diagnostic test. Differences between the predicted and the measured signal can be eliminated by the medical practitioner in an iterative process by modifying the morphological data of the model lung. These modifications support the diagnosis by localizing the obstructions in the airway tree and by quantifying the severity of these obstructions.

\section{Methods}

The basis of the SBW model is a one-dimensional advection-diffusion equation,

$$
\frac{\partial q}{\partial t}=\hat{D} \frac{\partial^{2} q}{\partial x^{2}}-\hat{u} \frac{\partial q}{\partial x}+\alpha q
$$

with

$$
\hat{D}=\frac{S_{d} D}{S_{d}+S_{a}}, \quad \hat{u}=\frac{1}{S_{d}+S_{a}}\left[S_{d} u-D \frac{\partial S_{d}}{\partial x}\right],
$$

where $q \in[0,1]$ is the concentration of a certain gas species, $x$ is the spatial coordinate along the airway and $t$ is the time. The diffusion coefficient $D$ is a physical property of the gas and kept constant. In addition, Taylor diffusion is integrated into the model by an analytical approach. $S_{d}$ and $S_{a}$ are the cross-section of the duct and the alveolei respectively, based on data from [2]. The parameter $\alpha$ models the absorption of a gas into the tissue (e.g. oxygen uptake through alveolar membranes). For the inert gas species studied here we assume $\alpha=0$.

The advection velocity $u$ is calculated on the assumption of laminar flow in a bifurcating ductal network, the local flow resistance is based on the Poiseuille law. The volume flow at the mouth is prescribed as a sinusoidal function with given breath period and tidal volume.

In order to reduce the computational cost for solving the equations, we use a largely symmetric lung model which allows us to resolve only on the order of $N$ different airways rather than all $2^{N}$ airways in a lung (where $N$ is the number of airway generations).

The model described so far is closely related to a symmetric trumpet model. In an extension, we include asymmetric branches to determine inhomogeneous gas transport in the lung, based on localised ventilation differences in the hierarchical bifurcating duct flow. This is realised by adjusting the resistance at the branches and therefore the ventilation into the distal airways and acini. Changes are applied by assigning a transmissibility factor to the airway resistance, the lower the factor the higher the resistance. 
The simulation is programmed in Matlab, with a GUI as front end. Input parameters include the tidal volume (TV), functional residual capacity (FRC) and breath period. The main usage of the GUI is to define the asymmetric structure of the lung and introduce restrictions. Figure 2 shows the GUI for a example case, where we introduced variations after generation 8 .

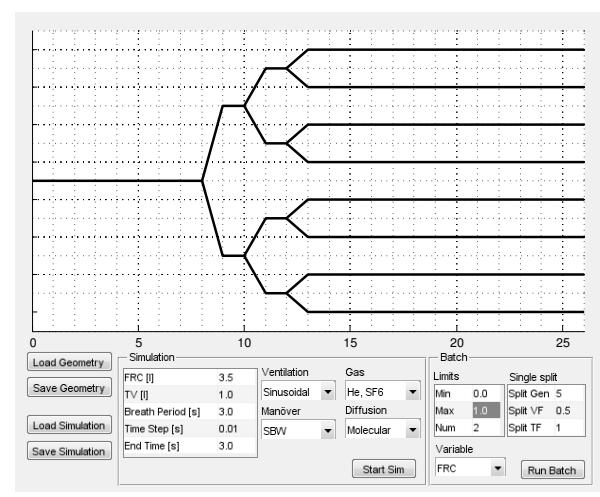

Figure 2: GUI of computer model.

In Figure 3 the resulting morphology is shown. Each branch splits into $20 \%$ and $80 \%$ of total sub-volume with one restricted daughter branch.

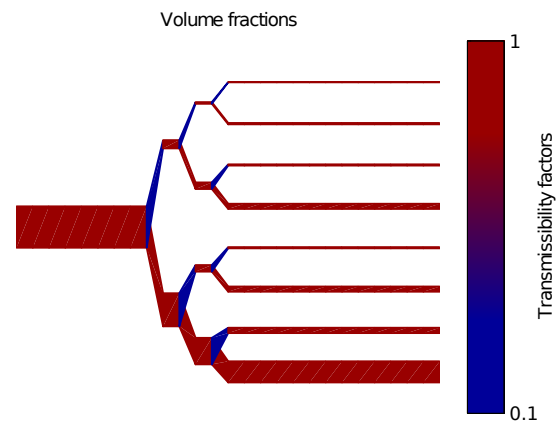

Figure 3: Visualization of the modelled lung morphology, the thickness of the bar depicts the fraction of the whole lung at that generation. The transmissibility factors are colour coded.

\section{Results}

As an example we present model results (figure 4) for a DTG-SBW test on the lung morphology shown in figure 3. The predicted MM signal (figure 5) can be compared to measured data (e.g. figure 1). In particular, the following characteristic values are compared:

$$
\begin{array}{ll}
\mathrm{MM}_{\max }: & \text { Maximum molar mass during exhalation } \\
\mathrm{V}_{\max }: & \text { Expired volume at maximum molar mass } \\
\mathrm{S}_{I I I}: & \begin{array}{l}
\text { Slope of MM signal (figures } 1 \text { and 5) during } \\
\text { phase III }
\end{array}
\end{array}
$$

Figure 5 illustrates how SIII depends on the severity of the obstructions in the fractal lung model of figure 3.

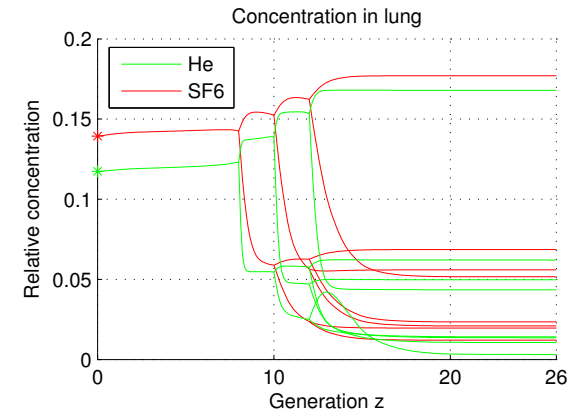

Figure 4: Simulated gas distribution for obstructed pathways.
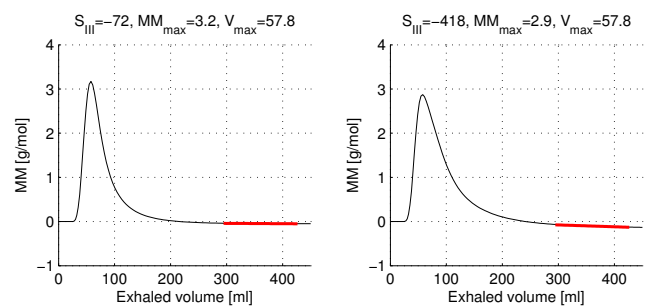

Figure 5: Concentration profile at mouth. Left: symmetric lung, $\mathrm{S}_{I I I}=-72 \mathrm{mg} / \mathrm{mol} / \mathrm{L}$. Right: obstructed lung, $\mathrm{S}_{I I I}=-418 \mathrm{mg} / \mathrm{mol} / \mathrm{L}$.

\section{Discussion}

In CF lung disease, steep SIII reflects ventilation inhomogeneity near the acinus entrance. The present DTGSBW is a promising test to assess ventilation inhomogeneity in an easy and fast way and has great potential for widespread clinical use. The integration of the computer model into available software will help understanding underlying pathophysiological processes. Before this can be done on a routine basis, further studies of the exact effect of different morphological asymmetries are required.

\section{Acknowledgement}

This study was supported by an unrestricted grant no. 14435.1 PFLS-LS of the Federal Department of Economic Affairs Switzerland, Commission for Technology and Innovation (CTI), Innovation Promotion Agency.

\section{Bibliography}

[1] F. Singer, G. Stern, C. Thamrin, C. Abbas, C. Casaulta, U. Frey, and P. Latzin, "A new double-tracer gas singlebreath washout to assess early cystic fibrosis lung disease," European Respiratory Journal, vol. 41, no. 2, pp. 339-345, 2013.

[2] D. P. J. Six, W. R. De Vries, and S. C. M. Luijendijk, "Alveolar sacs and the expirograms of He and SF6: a model study," Comput. Biomed. Res., vol. 24, pp. 321331, July 1991. 